\title{
Imipramine and Alprazolam Effects on Stress Test Reactivity in Panic Disorder
}

\author{
Walton T. Roth, Jürgen Margraf, Anke Ehlers, John M. Haddad, \\ Richard J. Maddock, W. Stewart Agras, and C. Barr Taylor
}

The reactivity of 40 panic disorder patients on mental arithmetic, cold pressor, and 5\% $\mathrm{CO}_{2}$ inhalation stressors was tested before and after 8 weeks of treatment with imipramine, alprazolam, or placebo. Mean levels of subjective and physiological stress measures were compared during a baseline before any stressors were given, and at anticipation, stressor, and recovery periods for each stressor. After treatment, imipramine patients differed from the other two treatment groups on the prestressor baseline in showing higher systolic blood pressure (mean difference about $10 \mathrm{~mm} \mathrm{Hg}$ ), higher diastolic blood pressure (10 $\mathrm{mm} \mathrm{Hg})$, higher heart rate (15 bpm), less respiratory sinus arrhythmia, shorter pulse transit time, and lower $T$-wave amplitude. Respiratory measures, electrodermal measures, body movement, and self-reported anxiety and excitement did not distinguish the groups. Reactivity to the stress tests was unaffected by the medications, but tonic differences present in the baseline persisted.

\section{Introduction}

Two drugs that have little in common chemically or in their side-effect profiles-the tricyclic antidepressant, imipramine, and the benzodiazepine, alprazolam-are both efficacious in treating panic disorder (Zitrin et al 1983; Sheehan et al 1980; Lydiard and Ballenger 1987; Ballenger et al 1988). Certain aspects of the biochemical and neurophysiological mechanisms by which these drugs affect anxiety are understood (Hsiao and Potter 1990), and some information is available on their actions at the psychophysiological levels in humans. Anxiolytic drugs would be expected to reduce the psychological and physiological impact of stressors, and effects of this sort have been demonstrated for certain benzodiazepines and certain populations (e.g., Kelly et al 1973; O'Boyle et al 1986; McLeod et al 1988, McLeod et al 1990). The last study compared imipramine and alprazolam in generalized anxiety disorder patients and found that after 6 weeks imipramine had increased heart rate, blood pressure, and electromyographic activity over baseline levels in spite of reducing somatic anxiety symptoms. Alprazolam had reduced systolic

From the Veterans Affairs Medical Center, Palo Alto, CA (WTR, JMH), the Department of Psychiatry and Behavioral Sciences, Stanford University School of Medicine, Stanford, CA (WTR, WSA, CBT), Fachbereich Psychologie, Philipps-Universitat Marburg, Marburg/Lahn, Germany (JM, AE), and the Department of Psychiatry, University of California Davis School of Medicine, Sacramento, CA (RJM).

Address reprint renguests to Walton T. Roth, M.D., Psychiatry 116A3, VA Medical Center, 3801 Miranda Avenue, Palo Alto. CA 94304.

Received March 6, 1991; revised July 22, 1991. 
blood pressure and somatic symptoms in the same period. Unfortunately, the absence of a placebo group made it impossible to separate drug effects from time effects. In other experiments, certain benzodiazepines and barbiturates were shown to increase reliably the rate of decrease of autonomic arousal in anxious patients sitting quietly in a laboratory and to accelerate habituation to moderately intense tone stimuli (Lader and Wing 1966).

The effect of imipramine on cardiovascular stress response is particularly important because of its tendency to alter heart rate and blood pressure (reviewed recently by Jefferson 1989; Roose and Glassman 1989; Warrington et al 1989). It greatly elevates heart rate in subjects standing, sitting, lying awake, and asleep (Taylor et al 1990; Roth et al 1988). Although most clinicians are aware of imipramine's tendency to produce orthostatic hypotension, fewer realize that under certain conditions imipramine can raise systolic and diastolic blood pressure (Warrington et al 1989; Taylor et al 1990; McLeod et al 1990). Because panic disorder patients may be at increased risk for cardiovascular death (Coryell et al 1982, 1986), blood pressure elevations in this group are particularly undesirable. The effects of mental stressors on imipramine's cardiovascular effects in panic disorder patients is unknown: imipramine might increase blood pressure reactivity to stress, adding to its cardiovascular risk, or it might dampen reactivity through its anxiolytic or physiological actions.

The current study compared the effects of imipramine, alprazolam, and placebo on the reactivity to three laboratory stressors-mental arithmetic, ice water, and $\mathrm{CO}_{2}$ inhalation-in panic disorder patients and matched normal controls. The first two are stressors not suspected of having any special panic-inducing properties, while the last has been postulated to trigger specific panic mechanisms (Gorman et al 1984). The design of the study allowed us to measure baseline levels, reactivity, and recovery of a number of cardiovascular and electrodermal variables associated with autonomic activation and to compare them with subjective reports of moods and symptoms and with behavior. Eysensk (1967) characterized "neurotics" as generally more reactive autonomically than nonneurotics. More recently, hyperexcitablity of brainstem loci controlling the autonomic nervous system has been postulated as a cause for panic attacks (Gorman et al 1989). Therefore, we expected autonomic reactivity to be greater in panic patients than in normals, and that this hyperreactivity would be normalized by effective treatment. We also assessed ventilatory response to $\mathrm{CO}_{2}$ since that has been reported to differ between panic patients and normals (Gorman et al 1988; Lousberg et al 1988; Papp et al 1989).

Special care was taken to exclude patients who were surreptitiously taking anxiolytic drugs not prescribed to them (Clark et al 1990). Elsewhere (Roth et al 1991) we reported pretreatment differences between a larger group of patients and normal subjects, of which the sample here is a subset, and the effects of treatment on psychological outcome, plasma lipids, ambulatory heart rate, and blood pressure and heart rate during treadmill exercise (Taylor et al 1990).

\section{Methods}

\section{Subjects}

Eighty-six patients with panic disorder were recruited through the media for a study comparing the effects of imipramine, alprazolam, and placebo, and participated in the testing protocol described below at the week 0 pretreatment laboratory test session. As determined by the Structured Clinical Interview of DSM-III Upjohn Version (SCID-UP) 
Table 1. Subject Characteristics ${ }^{a}$

\begin{tabular}{lcccc}
\hline & & Patients & & \\
& Imipramine $^{b}$ & Alprazolam & Placebo & Normals \\
\hline Number & 13 & 14 & 13 & 14 \\
Male & 5 & 4 & 4 & 4 \\
Age (yr) & $33.0(5.8)$ & $36.4(8.1)$ & $31.4(10.3)$ & $33.2(8.6)$ \\
Pills $(n)$ & $5.5(1.6)$ & $4.0(2.3)$ & $7.3(1.7)$ & - \\
Blood levels (ng/ml) & $83.3(47.7)$ & $58.2(46.2)$ & $0.0(0.0)$ & - \\
\hline
\end{tabular}

${ }^{a}$ Results given as mean \pm SD.

${ }^{b}$ Blood levels for the imipramine group are the sum of imipramine and desmethylimipramine.

(Spitzer and Williams 1983), all met the panic attack criteria of DSM-III Panic Disorder, although some qualified for a DSM-III diagnosis of Agoraphobia with Panic Attacks because of their extensive avoidance. Additionally, patients had to have had at least one attack in each of the 3 weeks before entering the study. Any major depressive episodes in the present or past needed to follow and not precede the initial occurrence of panic attacks. Patients had to be in good health as determined by medical history, physical examination, electrocardiogram (ECG), and blood and urine tests. They were told that they must stop taking psychoactive drugs 2 weeks before testing, and blood was drawn and assayed for diazepam, desmethyldiazepam, alprazolam, imipramine, and desmethylimipramine at the laboratory session. For several reasons (for example, 17 had detectable levels of the compounds mentioned above), the data from 34 were excluded from analysis, leaving 52 at week 0 (for further details see Roth et al 1991).

Patients were randomly assigned to 8 weeks of treatment with imipramine, alprazolam, or placebo, and were tested again in the laboratory during the 8th treatment week. Identical capsules containing imipramine $30 \mathrm{mg}$, alprazolam $1 \mathrm{mg}$, or placebo were administered double-blind. Medication dose was increased gradually until patients were free of panic attacks or were taking 10 capsules per day. At week 8, a subset of 40 patients of the 52 patients was selected for statistical analysis. These $\mathbf{4 0}$ patients were those who returned for week 8 laboratory testing, whose blood drawn at week 8 contained the proper compounds, and who could be matched to create medication groups that were age and sexmatched. Table 1 summarizes characteristics of these patients and 14 matching normals. Average daily dose for imipramine was $165 \mathrm{mg}$ and for alprazolam, $4 \mathrm{mg}$.

Normals were recruited by newspaper advertisement. They were screened over the telephone and in structural interviews. They all described themselves as "nonanxious" and did not have a history of psychiatric problems as determined by the SCID-UP and a structured interview based on the SADS-L (Spitzer and Endicott 1978). Anyone with significant phy'sical health problems was rejected. Normals were tested twice at about the same interval as the patients, but were not given medication.

\section{Procedure}

Testing took place in a sound-attenuated chamber in which subjects could not see laboratory personnel but could communicate with them at any time. Four test paradigms were administered: a 15-min baseline, cold pressor (ice water), mental arithmetic, and $\mathrm{CO}_{2}$ inhalation. Written instructions for each were read by the subject just prior to the first period of that test. Subjects were told they could ask that a test be discontinued at any time, but were 
encouraged not to do so. The beginning and end of each test period was announced by the experimenter through an intercom. The baseline paradigm simply required subjects to sit with their eyes open for $15 \mathrm{~min}$. The cold pressor tesî consisted of a 4-min anticipation period, 1 min during which the subject's dominant foot was immersed in ice water $\left(4^{\circ} \mathrm{C}\right)$, and $7 \mathrm{~min}$ of recovery. The mental arithmetic test consisted of a 3-min 45-sec anticipation period, a 5-min 15-sec period of spoken serial subtractions of 17 from 5797, and a 7-min recovery period. For the $\mathrm{CO}_{2}$ inhalation test, subjects wore a continuous positive-pressure gas mask. For the first 15-min anticipation period they breathed compressed room air, which was switched secretly in an adjacent room to $5.5 \% \mathrm{CO}_{2}$ in room air for $20 \mathrm{~min}$. Then the gas was switched back to room air for a 15 -min recovery period. Continuous monitoring with a capnometer insured that inhaled air during the $\mathrm{CO}_{2}$ period had a $\mathrm{CO}_{2}$ concentration above $5 \%$. Although subjects were blind to when $\mathrm{CO}_{2}$ began, they were told when it was stopped. The $\mathrm{CO}_{2}$ seemed to have a different smell than room air, so as to increase the subjects' uncertainty about when they were receiving $\mathrm{CO}_{2}$, the experimenter switched from air to $\mathrm{CO}_{2}$ just long enough for one breath at minutes 4 and 9 , and from $\mathrm{CO}_{2}$ to air at minutes 19,24 , and 29. The baseline paradigm was always first, and the $\mathrm{CO}_{2}$ inhalation, always last since we thought that it might cause subjects to terminate the session. The order of cold pressor and mental arithmetic was balanced across subjects.

\section{Physiological Recording}

At the beginning of the session, a pair of $\mathrm{Ag} / \mathrm{AgCl}$ electrodes $0.8 \mathrm{~cm}^{2}$ in area was placed on the thenar and hypothenar eminences of the nondominant hand for recording skin conductance. The skin conductance transducer applied a constant $0.5 \mathrm{~V}$ across the electrodes. The electrode medium was a mixture of creamy ointment and physiological saline as recommended by Fowles et al (1981). An ECG was recorded from electrodes over the left 10th rib at the anterior axillary line and the right mastoid. A photoreflective skin blood flow transducer was put on the nondominant thumb (T23-04, Coulbourn Instruments, Lehigh Valley, PA). Impedance registration of changes in blood volume during the cardiac cycle was made from electrodes on the dominant forearm, one measurement electrode placed on the dorsum of the forearm slightly proximal to the wrist and the other slightly distal to the elbow. Two elastic belts were placed over the rib cage and abdomen, respectively, and were connected to a device that measures chest movement during respiration by changes in selfinductance (Respitrace Corporation, Ardsley, NY). This device was calibrated for respiratory volume by having subjects breathe into a spirometer. Body movements in the chair were monitored piezoelectrically with a phonograph cartridge mounted in the seat.

Systolic and diastolic blood pressure were measured oscillometrically by an automatic device (Accutorr 2, Datascope Corporation, Paramus, NJ). The first blood pressure measurement was $60 \mathrm{sec}$ after the start of each paradigm and then every 4.5 min during the baseline, 2 min during the cold pressor test (including a measurement at the end of immersion) and mental arithmetic tests, and every 2.5 min during $\mathrm{CO}_{2}$ inhalation. Inflation of the cuff, which was placed on the nondominant arm, caused large changes in finger blood flow registered by the device described above.

\section{Psychological Assessment}

Subjects filled out an Anxiety Rating Scale (Ehlers et al 1986) right after each deflation of the blood pressure cuff. Subjects rate themselves on "anxiety" and "excitement," each 
on a scale from 0 ("none") to 10 ("extreme"). Additional assessments were given to evaluate possible panic attacks, but since only one subject reported a panic attack at week 8 , these assessments are not described further here.

Clinical self-assessments and physician assessments of the patients were made at weeks 0 and 8. Details about these assessments and the results for a larger sample that included the same patients reported here can be found in Taylor et al (1990) and Clark et al (1990). Representative clinical measures analyzed for our subgroup included average daily anxiety (0 to 10 scale) for the last week recorded in a diary, Hamilton Rating Scale for Anxiety scores, number of panic attacks/week, Symptom Checklist-90 (SCL-90) anxiety scores, and patient-rated Global Improvement since beginning medication $(1=$ very much better, $4=$ no change, 7 = very much worse).

\section{Data Reduction and Statistical Analysis}

Continuously recorded physiological data were digitized and stored on tape for off-line analysis. Sampling rates were $200 \mathrm{~Hz}$ for the electrocardiographic, plethysmographic, and body movement channels and $20 \mathrm{~Hz}$ for skin conductance and respiration. Cardiovascular variables included heart rate calculated from $R-R$ intervals, $T$-wave amplitude, finger pulse amplitude calculated as the difference between maximum and minimum levels during each cardiac cycle, and pulse transit times calculated from $\mathbf{R}$ waves to maxima in the forearm impedance channel and in the finger blood flow channel. Nonspecific skin conductance fluctuations were defined as an increase in skin conductance level (SCL) of 0.05 microsiemens occurring within $3.5 \mathrm{sec}$ after a change in SCL slope of at least 0.01 millisiemens/sec from a positive or negative value. Respiratory rate, and minute volume were calculated from the respiratory volume curves using a computer algorithm. Respiratory sinus arrhythmia was quantified by measuring the mean difference in $\mathbf{R}-\mathbf{R}$ interval between inspiratory and expiratory phases of the breathing cycle (Grossman and Wientjes 1986). Body movement was quantified as the integral of the absolute values of the transducer output around its mean.

Statistical analyses were based on mean values of purely cardiovascular variables over 10-sec epochs, and mean values of other variables over 30-sec epochs. Data were edited to exclude sensor and motion artifacts and periods when the blood pressure cuff was inflated. Repeated-measures analysis of variance (ANOVA) with statistical significance levels adjusted according to the Greenhouse-Geisser correction was a principal statistical method. Subanalyses of variance and post hoc comparison of means (using Tukey's Studentized Range Test corrected for unequal sample sizes) were applied to localize the source of effects significant in more global analyses.

Data from the baseline paradigm were averaged into three 5-min epochs and analyzed in a two-way analysis of variance with Group (imipramine, alprazolam, placebo patients or normals, placebo patients) as one factor and Time (epochs 1, 2, and 3) as a second, repeated-measures, factor. Data from the stress paradigms were averaged into prestressor (anticipatory), stressor, and poststressor (recovery) periods for each stressor, and a threeway repeated-measures ANOVA was performed. Factors were Stressor (cold pressor, arithmetic, $\mathrm{CO}_{2}$ ), Time (prestressor, stressor, poststressor), and Group (imipramine, alprazolam, placebo patients or normals, placebo patients). Stressor and Time were repeated measures. All ANOVAs were done on both week 0 data and on week 8-week 0 differences between corresponding data points of each subject. The latter analysis is more sensitive to changes produced oy drug treatments than an analysis of week $\mathbf{8}$ data alone. Following 
the recommendations of Venables and Christie (1980), SCL was logarithmically transformed prior to statistical analysis. Degrees of freedom varied in different analyses because or missing data. For example, subjects who refused $\mathrm{CO}_{2}$ at week 8 were not included in the stress paradigm ANOVA. Pulse transit time measurements were missing for certain subjects. For subjects who stopped $\mathrm{CO}_{2}$, that stress period contained fewer data and was biased towards earlier measurements.

ANOVAs on clinical measures except Global Improvement were also done on week 0 data and on week 8-week 0 differences. Global Improvement was analyzed for week 8 data alone, since patients were asked then to rate change since beginning medication.

Results

\section{Pretreatment Differences}

Because patients were randomly assigned to drug groups, no pretreatment differences among imipramine, alprazolam, and placebo patients at week 0 would be expected. At week 0, five imipramine, three alprazolam, and three placebc patients asked to stop $\mathrm{CO}_{2}$ early; of these three, two, and two, respectively, gave panic attacks as the reason for stopping.

Figures 1 and 2 depict the week 0 means of patients in the three drug groups for the baseline and stress paradigms. ANOVAs of the baseline paradigm showed no significant main Group effects, but by chance, Time $\times$ Group interactions were significant for respiratory sinus arrhythmia $[F(4,74)=3.46, \varepsilon=0.754, p<0.05]$ and respiratory rate $[F(4,74)=3.54, \varepsilon=0.854, p<0.05]$. Although not significant, $\mathrm{T}$-wave amplitude tended to be higher and heart rate lower in the imipramine group, while the means of variables like systolic and diastolic blood pressure and pulse transit time were closely matched. ANOVAs of the stress paradigms showed no Group effects or Group interactions. There were significant Time (prestressor, stressor, poststressor) effects for all variables, indicating that the variables selected were sensitiv to al least one of the stressors employed. In addition, about half of the variables showed Stressor effects or Stressor $x$ Time interactions. Essentially identical effects in the larger pool of patients from which this sample is drawr. are discussed in Roth et al (1991). That article also compares normals and palints before treatment.

Tr'ile 2 gives the clinical measures at week 0 . None of these differed significantly between drug groups.

\section{Treatment Effects}

At week 8 refusals and requests to stop the stressors were limited largely to patients on placebo or imipramine during $\mathrm{CO}_{2}$ (six patients on placebo, four on imipramine, two on alprazolam, and one normal). Of these, two patients on placebo, two on imipramine, one on alprazolam, and one normal refused $\mathrm{CO}_{2}$ altogether. However, the differences between the four groups during $\mathrm{CO}_{2}$ did not reach significance $\left(\chi^{2}=6.87 ; p=0.08\right)$. Differences among the three patient groups were also nonsignificant $\left(\chi^{2}=3.27 ; p=\right.$ 0.20 ). Only one subject, a patient on placebo, gave panic attack as a reason for stopping $\mathrm{CO}_{2}$. The only other stress test not completed was cold pressor, which was refused by a normal.

Figures 3 and 4 show the mean week 8 -week 0 changes of patients in the three drug groups for the baseline and stress paradigms. Table 3 gives the ANOVA significance 


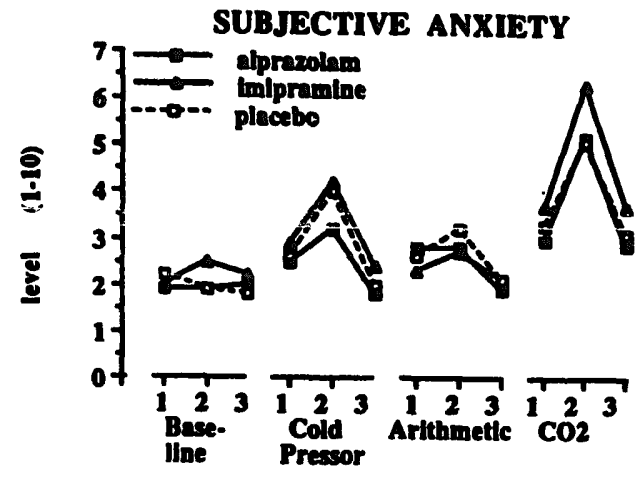

SUBJECTIVE EXCITEMENT

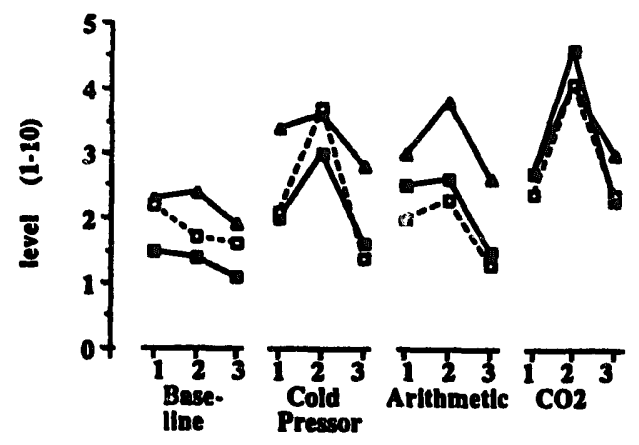

HEART RATE

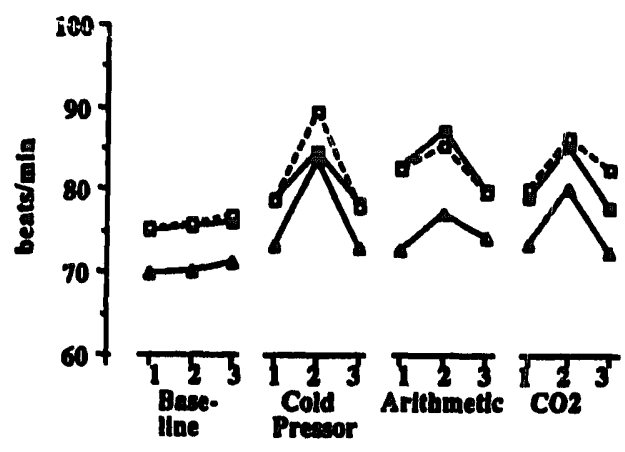

RESPIRATORY SINUS ARRHYTHMI

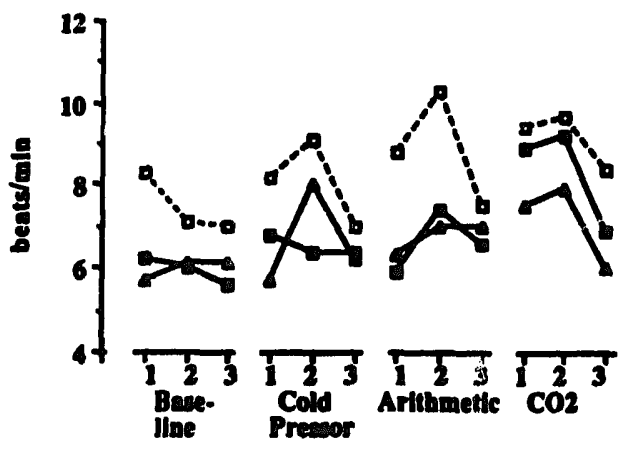

SYSTOLIC BLOOD PRESSURE

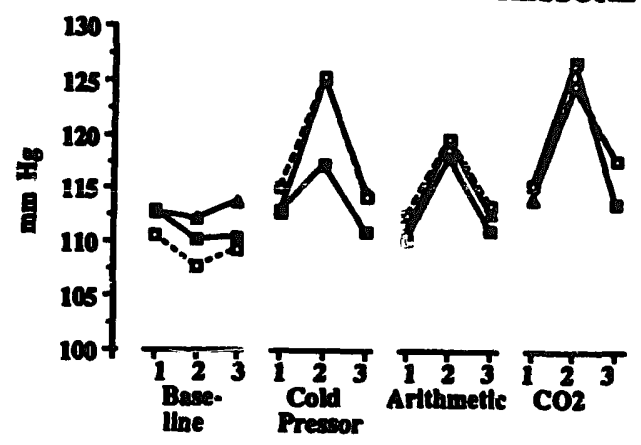

DIASTOLIC BLOOD PRESSURE

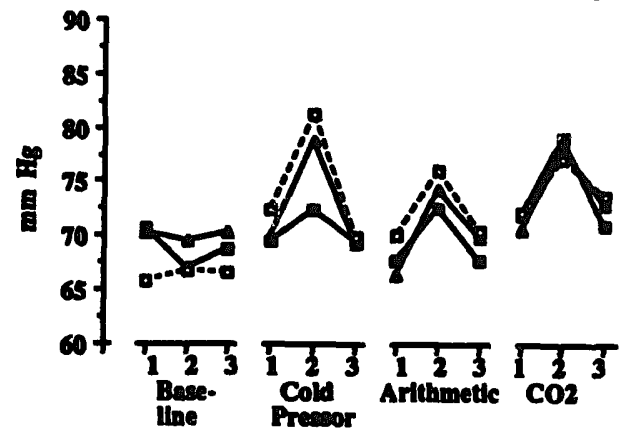

PULSE TRANSIT TIME

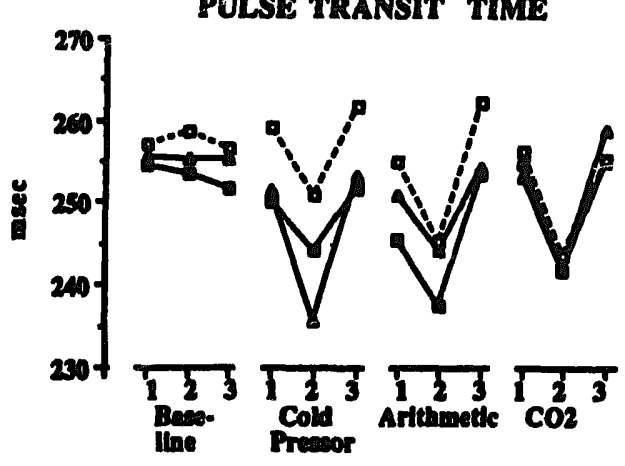

T-WAVE AMPLTUDE

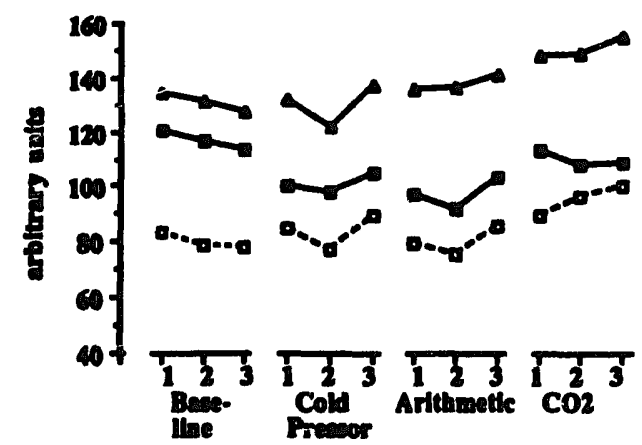

Figure 1. Week 0 means for imipramine, alprazolam, and placebo patients. The baseline is divided into three 5 -min time periods $(1,2$, and 3$)$ and each of the stress paradigms is civided into prestressor (anticipation), stressor, and poststressor (recovery) periods (1, 2, and 3). 
MINUTE VOLUME

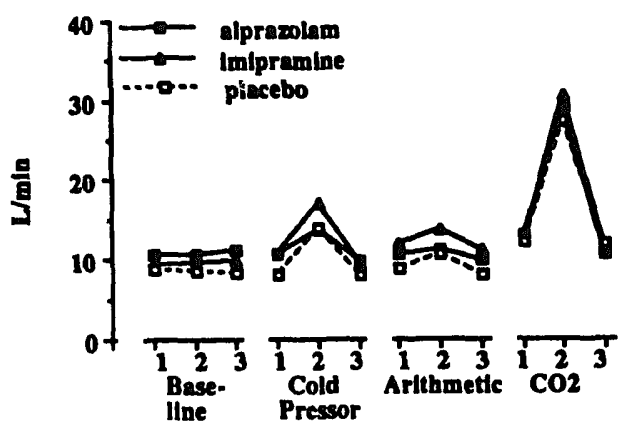

RESPIRATION RATE

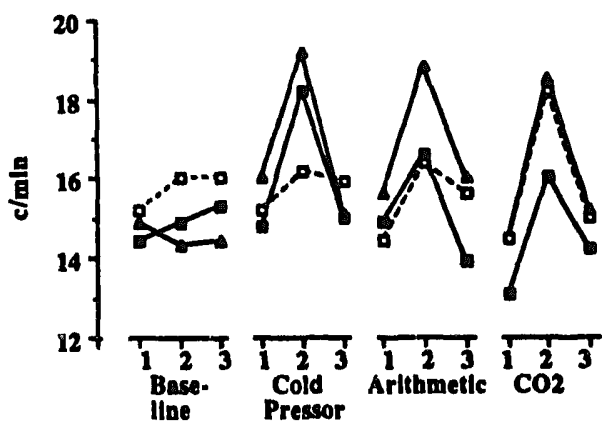

BODY MOVEMENT

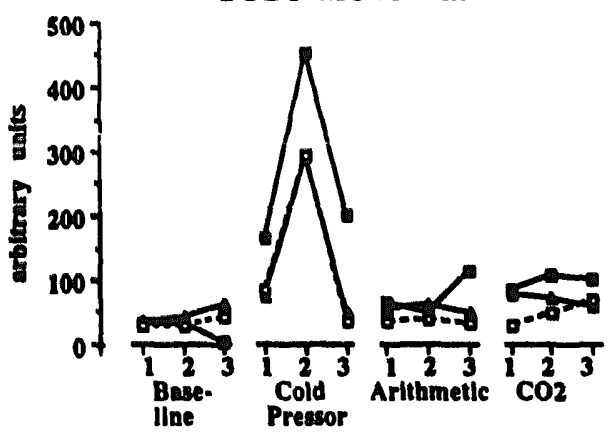

FINGER PULSE AMPLITUDE

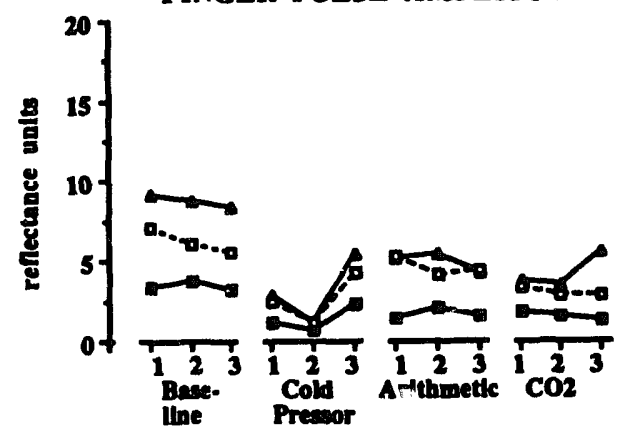

SKIN CONDUCTANCE LEVEL

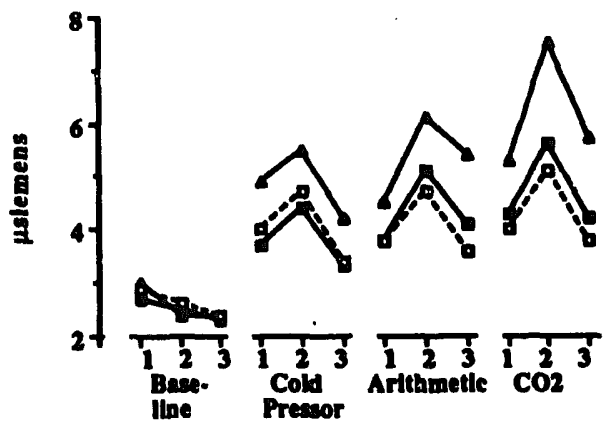

SKIN CONDUCTANCE FLUCTUATIONS

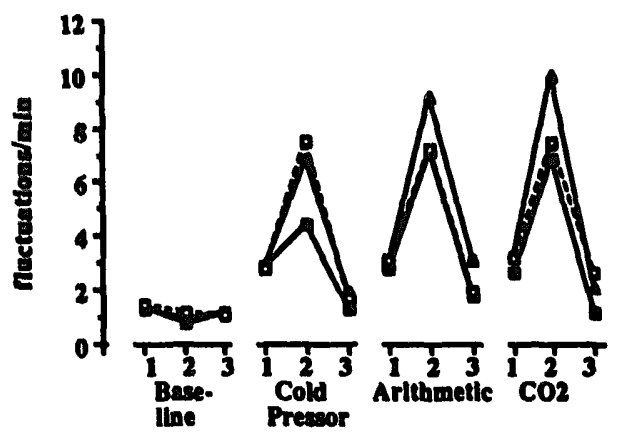

Figure 2. See Figure 1.

levels for the baseline paradigm. Group effects were significant for heart rate, systolic and diastolic blood pressure, respiratory sinus arrhythmia, pulse transit time, and T-wave amplitude. The only other significant effect was that of Time for respiratory rate, which apparently reflects a tendency for the difference in week 8 and week 0 rates to increase over time. Table 4 gives the group contrasts for variables with significant main Group effects in Table 3. Imipramine differed significantly from placebo in all six cases, and from alprazolam in four cases. For the imipramine group, intercorrelations were calculated between age and week 8 -week 0 changes in subjective anxiety, heart rate, systolic and diastolic blood pressure, respiratory sinus arrhythmia, pulse transit time, $T$-wave amplitude, and serum blood drug concentrations of imipramine plus desmethylimipramine. The change scores were based on variable means over all three time periods of the baseline 
Table 2. Clinical Outcome Measures ${ }^{a}$

\begin{tabular}{lcc}
\hline & Week 0 & Week 8-Week 0 \\
\hline Mean daily anxiety & & \\
Imipramine & $4.1(1.9)$ & $-2.3(1.4)$ \\
Alprazolam & $3.7(1.8)$ & $-1.7(1.7)$ \\
Placebo & $4.0(2.1)$ & $-1.3(2.1)$ \\
Hamilton anxiety & $13.0(5.4)$ & $-7.4(5.4)$ \\
Imipramine & $19.9(9.7)$ & $-14.1(10.4)$ \\
Alprazolam & $16.4(6.1)$ & $-9.0(5.5)$ \\
Placebo & & $-6.4(5.9)$ \\
Panic attacks & $6.6(6.1)$ & $-5.4(4.7)$ \\
Imipramine & $5.9(4.6)$ & $-5.7(4.7)$ \\
Alprazolam & $6.7(4.2)$ & $-7.3(5.8)$ \\
Placebo & $10.3(5.5)$ & $-10.3(10.6)$ \\
SCL-90 anxiety & $16.2(9.9)$ & $-5.7(5.3)$ \\
Imipramine & $14.0(5.8)$ & $1.5(0.66)$ \\
Alprazolam & - & $1.8(0.93)$ \\
Placebo & - & $3.1(1.2)$ \\
Global improvement & - & \\
Imipramine & Alprazolam &
\end{tabular}

${ }^{\top}$ Results given as mean \pm SD.

paradigm. The only significant correlations $(\mathrm{df}=11)$ were between systolic and diastolic blood pressure change $(r=+0.59, p<0.05)$. Correlations of blood pressure change with age $(r=-0.12)$ or serum levels $(r=-0.11)$ were far from significant.

Table 5 gives the significance levels for the stress paradigms. Main Group effects without interactions were significant for the same variables that showed Group effects for the baseline paradigm, and additionally, for movement. Table 6 gives the gioup contrasts for stress paradigm variables with significant main Group effects. Imipramine differed from both alprazolam and placebo for heart rate and systolic and diastolic blood pressure, respiratory sinus arrhythmia, and $\mathrm{T}$-wave amplitude differed only between imipramine and placebo, and pulse transit time differed only between imipramine and alprazolam. The main Time effects and the Stressor $\times$ Time effect were apparently due to a tendency for stressors to produce smaller effects at week 8 than at week 0 . Note that changes in either reactivity or recovery between week 0 and week 8 could give rise to Time effects in week 8-week 0 difference scores; the V-shaped patterns in Figure 3 during stress paradigms seen, for example, in systolic and diastolic blnod pressure and respiration rate, indicate decreased reactivity. Reactivity or recovery differences between drug groups would have resulted in Time $\times$ Group or Time $\times$ Group $\times$ Stressor effects, but only skin conductance fluctuations showed such effects. Although this variable showed a significant Time $\times$ Group interaction, no significant Group effects appeared in subanalyses for prestressor, stressor, and poststressor periods. Thus drug treatment had little or no effect on reactivity or recovery.

As shown in Table 2, all clinical measures showed improvement between week 0 and week 8. This improvement over time was statistically significant for all measures with all drug treatments except mean daily anxiety with placebo. However, only Global Improvement was differentially affected by the drugs $[F(2,35)=9.23, p<0.006]$. Post 


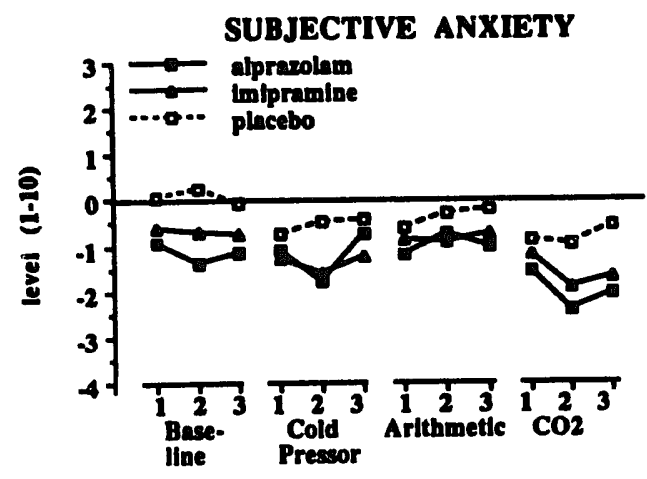

SUBJECTIVE EXCITEMENT
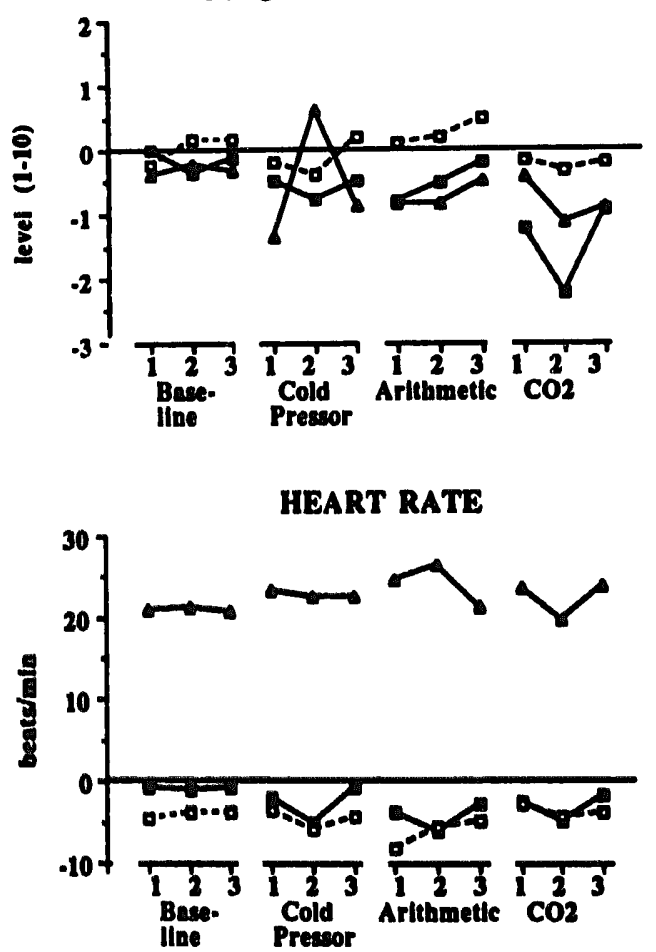

RESPIRATORY SINUS ARRHYTHMIA

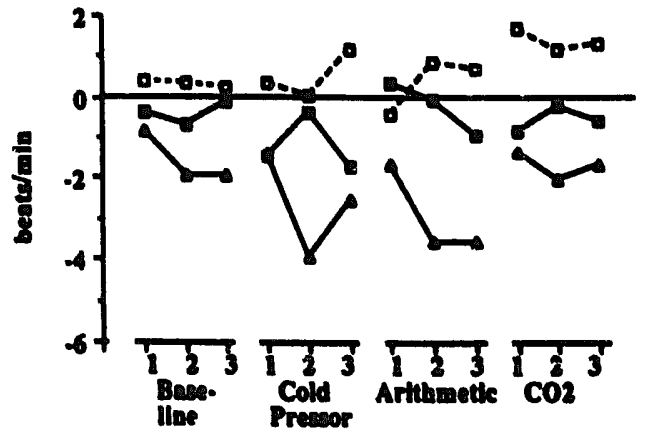

SYSTOLIC BLOOD PRESSURE

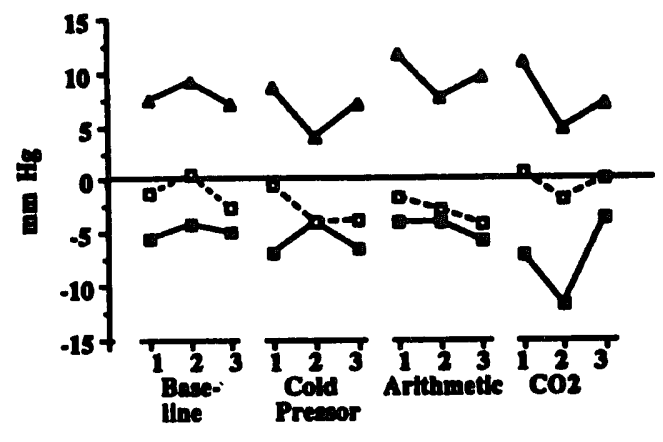

DIASTOLIC BLOOD PRESSURE

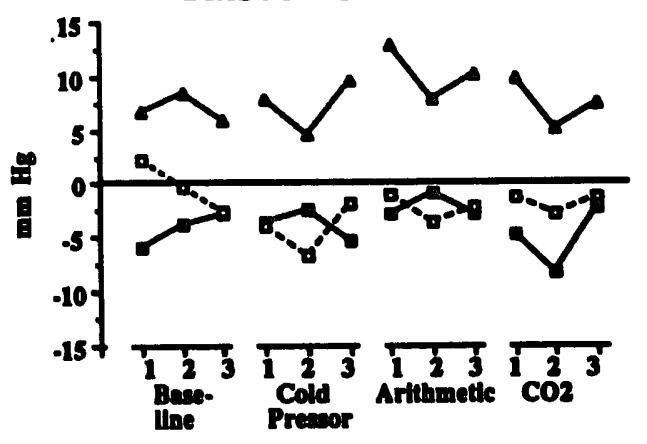

PULSE TRANSTT TIME

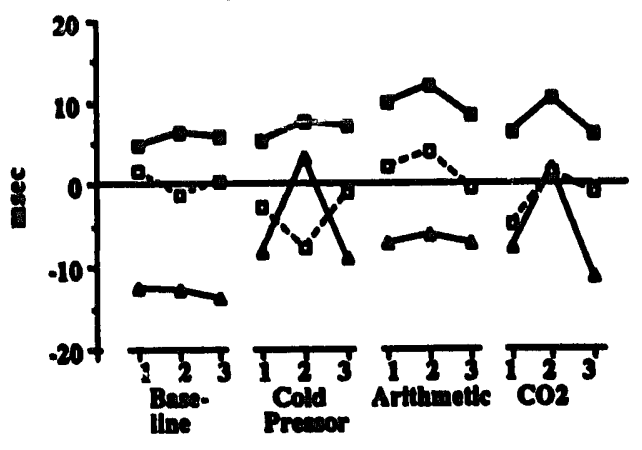

\section{T-WAVE AMPLITUDE}

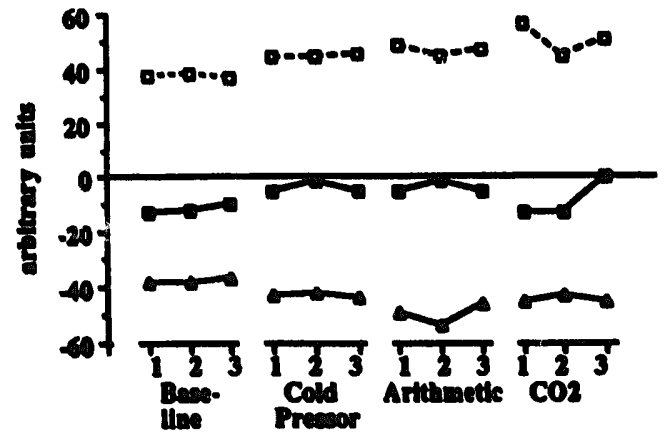

Figure 3. Week 8-week 0 mean changes for imipramine, alprazolam, and placebo patients. The baseline is divided into three 5 -min time periods $(1,2$, and 3$)$ and each of the stress paradigms is divided into prestressor, stressor, and poststressor periods (1,2, and 3). 

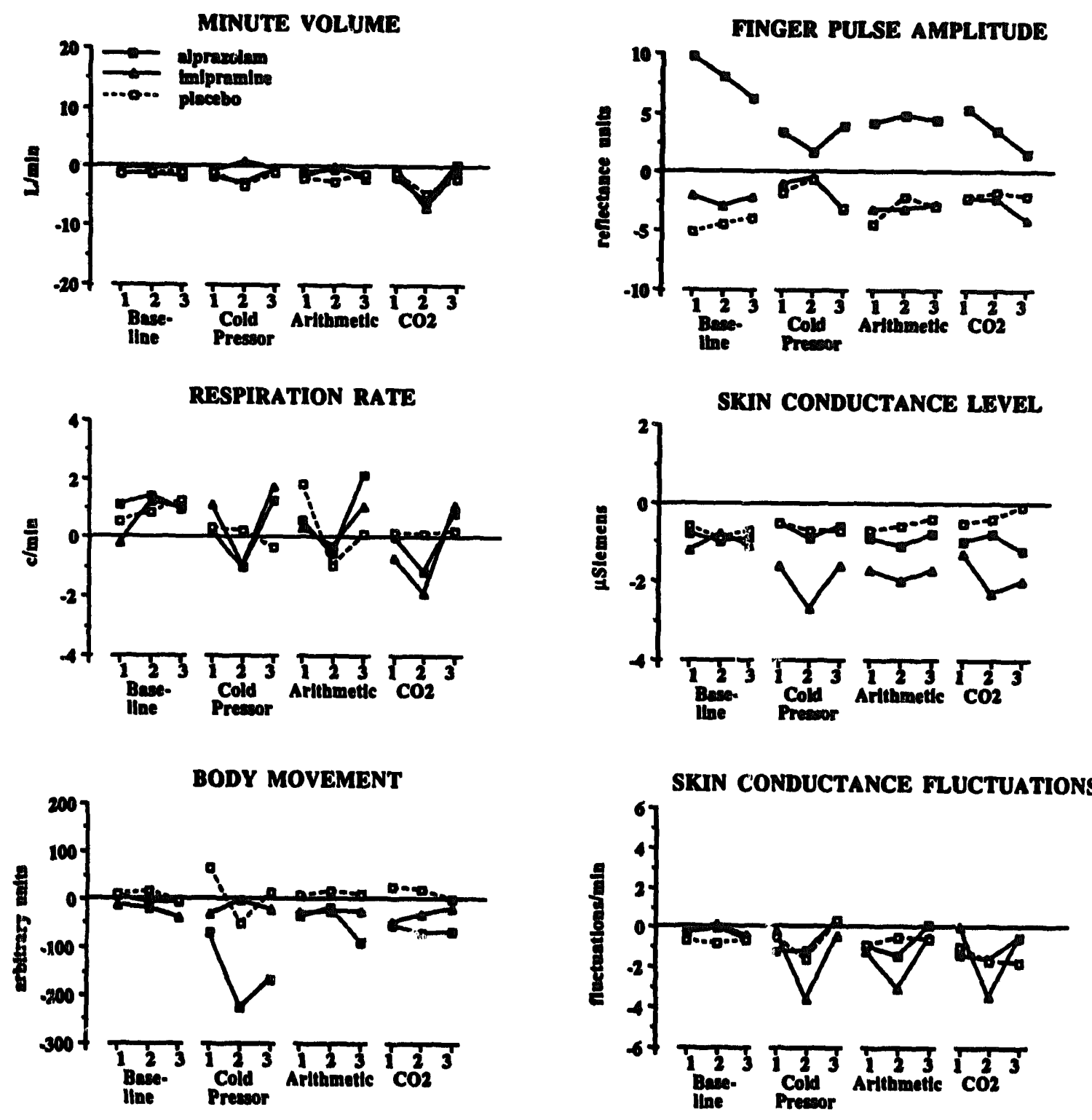

Figure 4. See Figure 3.

hoc comparisons showed that both imipramine and alprazolam were superior to placebo (lower scores on this measure indicate more improvement), but were not different from each other. There was a trend for alprazolam to lower Hamilton anxiety more than the other drugs [the overall $F(2,36)=2.85, p<0.07$ ].

\section{Comparison of Normals and Placebo Patients at Week 8}

ANOVAs of the baseline and stress paradigms for normals and placebo patients at Week 8 gave a gauge of whether patients not treated with an active medication but remaining in the study for $\mathbf{8}$ weeks differed from normal controls at that point in time (differences between normals and patients at week 0 are reported in Roth et al 1991). ANOVAs of 
Table 3. Significance Levels for Baseline Change (Week 8-Week 0): Imipramine, Alprazolam, and Placebo Patient: $s^{a, b, c}$

\begin{tabular}{|c|c|c|c|}
\hline Variable & T(ime) & G(roup) & TG \\
\hline \multicolumn{4}{|l|}{ Self-report } \\
\hline Anxiety & - & - & - \\
\hline Excitement & - & - & - \\
\hline \multicolumn{4}{|l|}{ Cardiovascular } \\
\hline Heart rate & - & $F(2,37)=37.2^{* * *}$ & - \\
\hline Systolic BP & - & $F(2,37)=14.3^{* * *}$ & - \\
\hline Diastolic BP & - & $F(2,37)=15.0^{* * *}$ & - \\
\hline RSA & - & $F(2,37)=3.94^{*}$ & - \\
\hline PTT & - & $F(2,25)=15.3^{* * *}$ & - \\
\hline FPA & - & - & - \\
\hline TWA & - & $F(2,37)=6.75^{* *}$ & - \\
\hline \multicolumn{4}{|l|}{ Electrodermal } \\
\hline SCL & - & - & - \\
\hline NSF & - & - & - \\
\hline \multicolumn{4}{|l|}{ Respiratory } \\
\hline Resp rate & $F(2,74)=3.93^{*}$ & - & - \\
\hline Minute voll & -- & - & - \\
\hline Movement & - & - & - \\
\hline
\end{tabular}

aProbability levels have been corrected according to Greenhouse and Geisser.

${ }^{b} \mathrm{BP}=$ blood pressure, $\mathrm{RSA}=$ respiratory sinus arrhythmia, $\mathrm{PTT}=$ pulse transit time, $\mathrm{FPA}=$ finger pulse amplitude, TWA $=\mathrm{T}$-wave amplitude, $\mathrm{SCL}=$ skin conductance level, $\mathrm{NSF}=$ nonspecific skin conductance fluctuations, vol $=$ volume.

'Significance: --not significant: ${ }^{*} p<0.05 ;{ }^{* *} p<0.01 ; * * * p 0.001$.

the baseline paradigm showed patients to have more subjective anxiety than normals $[F(1,25)=6.39, p<0.05]$, less respiratory minute volume $[F(1,25)=7.05, p<$ 0.05 ], and a greater increase in heart rate over $15 \mathrm{~min}$ [Time $\times$ Group: $F(2,50)=10.3$, $\varepsilon=0.777, p<0.001$ ], accompanied by a greater decrease in respiratory sinus arrhythm:ia [Time $\times$ Group: $F(2,50)=4.34, \varepsilon=0.852, p<0.05$ ].

ANOVAs of the stress paradigms showed Time effects for the same variables as the ANOVA for the three drug groups, that is, the same sensitivity to the stressors was present. Main Group effects or Group interactions were significant for subjective excitement, heart rate, systolic blood and diastolic blood pressure, nonspecific skin conductance fluctuations, respiratory minute volume, and body movement ( $p s<0.05$ or less). Subanalyses of variance and comparisons of means showed that for patients compared to normals, heart rate, and systolic blood pressure w'ere significantly higher during the entire

Table 4. Group Contrasts for Baseline Change $e^{a, b}$

\begin{tabular}{lccc}
\hline Variable & Imip/Alpraz & Alpraz/Placebo & Imip/Placebo \\
\hline Heart rate & $* *$ & - & $* *$ \\
Systolic BP & $* *$ & - & $* *$ \\
Diastolic BP & $* *$ & - & $* *$ \\
RSA & - & - & $*$ \\
PTT & $* *$ & - & $* *$ \\
TWA & - & - & $* *$ \\
\hline
\end{tabular}

"Abbreviations as in Table 3.

'Significance: - not significant: ${ }^{*} p<0.05 ;{ }^{* *} p<0.01$. 
Table 5. Significance Levels for Stress Test Change (Week 8-Week 0): Imipramine, Alprazolam, and Placebo Patients ${ }^{a, b, c}$

\begin{tabular}{|c|c|c|c|c|}
\hline Variable & $\mathrm{T}$ (ime) & G(roup) & $S$ (tressor)T & TG \\
\hline \multicolumn{5}{|l|}{ Self-report } \\
\hline Anxiety & - & - & - & - \\
\hline Exsitement & - & - & - & - \\
\hline \multicolumn{5}{|l|}{ Cardiovascular } \\
\hline Heart rate & $F(2,60)=3.56^{*}$ & $F(2,30)=48.1^{* * *}$ & - & - \\
\hline Systolic BP & $F(2,52)=5.26^{*}$ & $F(2,26)=11.1 * * *$ & - & - \\
\hline Diastolic BP & $F(2,52)=4.69^{*}$ & $F(2,26)=21.1 * * *$ & - & - \\
\hline RSA & - & $F(2,29)=9.28 * * *$ & - & - \\
\hline PTT & $F(2,22)=4.19 *$ & $F(2,11)=9.05^{* *}$ & - & - \\
\hline FPA & - & - & - & - \\
\hline TWA & - & $F(2,29)=7.02 * *$ & - & - \\
\hline \multicolumn{5}{|l|}{ Electrodermal } \\
\hline SCL & $F(2,60)=4.00^{*}$ & - & - & - \\
\hline NSF & $F(2,60)=18.5^{* * *}$ & - & - & $F(4,60)=5.20^{* *}$ \\
\hline \multicolumn{5}{|l|}{ Respiratory } \\
\hline Resp Rate & $F(2,60)=12 \cdot 1^{* *}$ & - & - & - \\
\hline Minute Vol & $F(2,60)=4.87^{*}$ & - & $F(4,120)=7.46^{* *}$ & - \\
\hline Movement & - & $F(2,29)=3.46^{*}$ & - & - \\
\hline
\end{tabular}

No S, GS, or TGS effects were significant.

aProbability levels have been corrected according to Greenhouse and Geisser.

"Abbreviations as in Table 3.

'Significance: - not significant; ${ }^{*} p<0.05 ;{ }^{* *} p<0.01 ;{ }^{* * *} p<0.001$

$\mathrm{CO}_{2}$ paradigm (not just while $\mathrm{CO}_{2}$ was being given); during immersion of the foot in ice water, body movement was less. There was no evidence for greater patient reactivity to stressors per se. (The details of the analysis are available from the author on request.)

\section{Discussion}

One rationale for this study was to determine if therapeutically effective antipanic medication would normalize autonomic hyperreactivity to stressors in panic patients. However, the assumption that our untreated panic patients would be hyperreactive compared with normals unexpectedly proved to be false, as we have reported elsewhere (Roth et al 1991). This was the case for both nonspecific stressors like cold pressor and mental

Table 6. Group Contrasts for Stress Test Change ${ }^{a, b}$

\begin{tabular}{lccc}
\hline Variable & Imip/Alpraz & Alpraz/Placebo & Imip/Placebo \\
\hline Heart rate & $* *$ & - & $* *$ \\
Systolic BP & $* *$ & - & $*$ \\
Diastolic BP & $* *$ & - & $* *$ \\
RSA & $-* *$ \\
PTT & $* *$ & - & - \\
TWA & - & - & $* *$ \\
Movement & - & - & - \\
\hline
\end{tabular}

${ }^{a}$ Abbreviations as in Table 3.

'Significance: - not significant; ${ }^{*} p<0.05 ;{ }^{*} p<0.01$. 
arithmetic and for panic-specific $\mathrm{CO}_{2}$. Consistent with this, placebo patients did not show greater reactivity than normals at week 8 . Our failure to observe autonomic hyperreactivity may be an indication that panic disorder arises solely from the occurrence of internal triggering events without any heightened sensitivity to external stressors. Because panic disorder was not characterized by autonomic hyperreactivity, it is less surprising that neither imipramine nor alprazolam affected physiolngical or psychological reactivity to our laboratory stressors, as indicated by the absence of Time $\times$ Group (except for skin conductance fluctuations) or Time $\times$ Group $\times$ Stressor interactions.

A lack of drug influence on reactivity could also be attributed to the active drugs and placebo having equal anxiolytic actions. The equality is consistent with the clinical effects of these drugs at 8 weeks. Patient-reported daily anxiety from diaries, anxiety ratings from the Symptom Checklist-90, and number of panic attacks decreased significantly but equally for imipramine, alprazolam, and placebo from week 0 to week 8 . Because the doses of the active drugs were adequate, the lack of differential treatment effects may at least in part be attributed to substantial improvement in placebo patients who remained in the study for 8 weeks. Only Global Improvement indicated a superiority of the active medications.

Rather than altering reactivity, imipramine tonically raised systolic and diastolic blood pressure, and heart rate, and reduced respiratory sinus arrhythmia, pulse transit time, and T-wave amplitude during baseline and stress paradigms. Paradoxically, this pattern is like the one induced by stressors, although it was not accompanied by increased anxiety. Although it could be argued that the approximately 5 beats/min lower heart rate in the imipramine group at week 0 left more room for increase by some Law of Initial Values, a difference of this magnitude could hardly explain the 30 beats $/ \mathrm{min}$ difference at week 8. That tonic drug effects were present during stress paradigms was demonstrated by main Group effects in the absence of interactions. Contrast statistics proved that these effects were generally due to imipramine differing from both alprazolam and placebo. The pattern of physiological change induced by imipramine was consistent with sympathetic activation (pulse transit time and $\mathrm{T}$-wave amplitude decrease) and vagal inhibition (respiratory sinus arrhythmia decrease). Respiratory sinus arrhythmia decrease was also observed by Jakobsen et al (1984) and McLeod et al (1990), while Middleton et al (1988) reported no change in this variable. The magnitude of the effect on systolic blood pressure was about $10 \mathrm{~mm} \mathrm{Hg}$, which in the case of nondrug-induced hypertension in women would increase coronary risk more than smoking a pack of cigarettes daily, adding 35 $\mathrm{mg} / \mathrm{dl}$ to one's serum cholesterol level, or being 2.8 years older (Truett et al 1967). This assumes, of course, that a higher blood pressure produced by imipramine has the same consequence as one due to other causes.

A number of important questions about the clinical effects of imipramine on blood pressure were not answered by our data. First, we do not know if blood pressure is elevated during daily activities less stressful than being in a scientific laboratory. Twentyfour heart rate measurements show that imipramine-induced rises persist even during sleep (Taylor et al 1990), but ambulatory blood pressure monitoring will be needed to establish whether the imipramine effect on blood pressure is sustained in a like manner. Second, the effects of posture need to be taken into account: The measurements presented here were made in sitting subjects, but blood pressure can vary with postural position, particularly in patients taking imipramine. Systolic and diastolic blood pressures of our imipramine patients immediately prior to 8-week treadmill testing (at a different laboratory session) were $8 \mathrm{~mm}$ higher than at week 0 when lying supine, but only $2 \mathrm{~mm}$ higher 
when standing (Taylor et al 1990); alprazolam and placebo patients, on the other hand, tended to have decreased supine blood pressures on repeat testing. Third, we do not know how far our results generalize beyond panic disorder, as panic disorder patients may have specific alterations in neurotransmitter regulation (Brown et al 1988). There is evidence, however, that other groups react with blood pressure elevations when treated with imipramine, namely, patients with generalized anxiety disorder (McLeod et al 1990), hyperactive children (Greenberg and Yellin 1975), depressed children (Preskorn et al 1983), and enuretic boys (Lake et al 1979). Fourth, the relationship between imipramine dose and blood pressure needs to be elucidated. There is some indication that blood pressure elevations occur primarily with lower doses of imipramine (reviewed in Warrington et al 1989).

One obvious implication of our results is that blood pressure elevation should be added to other reasons why imipramine is less safe than other kinds of anxiolytics in panic disorder patients at risk for cardiovascular disease. This, however, can never be the sole consideration in selecting a treatment for panic patients. For example, imipramine has the advantage over benzodiazepines, especially short-acting ones, of being easy to taper and discontinue without immediate recurrence of anxiety (reviewed by King et al 1990). Furthermore, the effectiveness of imipramine as an antidepressant commends it for panic patients who are significantly depressed.

This research was supported by the Department of Veterans Affairs, National Institute of Mental Health grant MH 40188, and the Upjohn Company.

We thank Maya L. Kopell, who wrote the data acquisition programs and certain of the data reduction programs, Sylvia Davies, who tested the subjects and helped in the data analysis, Mine Sakiyama, who made the figures, and Dr. D.J. Greenblatt, who provided the serum assays.

\section{References}

Ballenger JC, Burrows GD, DuPont RL, et al (1988): Alprazolam in panic discrer and agoraphobia: Results from a multicenter trial. I. Efficacy in short-term treatment. Arch Gen Psychiatry 45:413422.

Brown S-L, Charney DS, Woods SW, Heninger GA, Tallman J (1988): Lymphocyte $\beta$-adrenergic receptor binding in panic disorder. Psychopharmacology 94:24-28.

Clark DB, Taylor CB, Roth WT, et al (1990): Surreptitious drug use by patients in a panic disorder study. Am J Psychiatry 147:507-509.

Coryell W, Noyes R, Clancy J (1982): Excess mortality in panic disorder: A comparison with primary unipolar depression. Arch Gen Psychiatry 39:701-703.

Coryell W, Noyes R, House JD (1986): Mortality among outpatients with anxiety disorder. Am J Psychiatry 143:508-510.

Ehlers A, Margraf J, Roth WT, et al (1986): Lactate infusions and panic attacks: do patients and controls respond differently? Psychiatry Res 17:295-308.

Eysenck HJ (1967): The Biological Basis of Personality. Springfield, IL: Thomas.

Fowles DC, Christie MJ, Edelberg R, Grings WW, Lykken DT, Venables PH (1981): Publication recommendations for electrodermal measurements. Psychophysiology 18:232-239.

Gorman JM, Askanazi J, Liebowitz MR, et al (1984): Response to hyperventilation in a group of patients with panic disorder. Am J Psychiatry 141:857-861.

Gorman JM, Fyer MR, Goetz R, et al (1988): Ventilatory physiology of patients with panic disorder. Arch Gen Psychiatry 45:31-39.

Gorman JM, Liebowitz MR, Fyer AJ, et al (1989). A neuroanatomical hypothesis for panic disorder. Am J Psychiatry 146:148-161. 
Greenberg LM, Yellin AM (1975): Blood pressure and pulse changes in hyperactive children treated with imipramine and methylphenidate. Am J Psychiatry 132:1325-1326.

Grossman P, Wientjes K (1986): Respiratory sinus arrhythmia and parasympathetic cardiac controi: Some basic issues concerning qualification, applications and implications. In Grossman P, Janssen KHL, Vaitl D (eds): Cardiorespiratory and Cardiosomatic Psychophysiology. New York: Plenum, pp 117-138.

Hsiao JK, Potter WZ (1990): Mechanisms of action of antipanic drugs. In Ballenger JC (ed): Clinical Aspects of Panic Disorder. New York: Wiley-Liss, pp 297-317.

Jakobsen J, Hauksson P, Vetergaard P (1984): Heart rate variation in patients treated with antidepressants. An index of anticholinergic effects? Psychopharmacology 84:544-548.

Jefferson JW (1989): Cardiovascular effects and toxicity of anxiolytics and antidepressants. J Clin Psychiatry 50:368-378.

Kelly D, Pik R, Chen D-N (1973): A psychological and physiological evaluation of the effects of intravenous diazepam. Br J Psychiatry 122:419-426.

King D, Nicolini H, de la Fuente JR (1990): Abuse and withdrawal of panic treatment drugs. Psychiatr Ann 20:525-528.

Lade: MH, Wing L (1966): Physiological Measures, Sedative Drugs, and Morbid Anxiety. Institute of Psychiatry, Maudsley Monographs No. 14. London: Oxford University Press.

Lake CR, Mikkelsen EJ, Rapoport JL, Zavadil AP, Kopin IJ (1979): Effect of imipramine on norepinephrine and blood pressure in enuretic boys. Clin Pharmacol Ther 26:647-653.

Lousberg H, Griez E, van den Hout MA (1988): Carbon dioxide chemosensitivity in panic disorder. Acta Psychiat Scand 77:214-218.

Lydiard RB, Ballenger JC (1987): Antidepressants in panic disorder and agoraphobia. $J$ Affective Disord 13:153-168.

McLeod DR, Hoehn-Saric R, Labib AS, Greenblatt DJ (1988): Six weeks of diazepam treatment in normal women: Effects on psychomotor performance and psychophysiology. J Clin Psychopharmacol 8:83-99.

McLeod DR, Hoehn-Saric R, Zimmerli WD, De Souza EB, Oliver LK (1990): Treatment effects of alprazolam and imipramine: Physiological versus subjective changes in patients with generalized anxiety disorder. Biol Psychiatry 28:849-861.

Middleton HC, Nutt DJ, Anastassiades P (1988): Evidence that imipramine-induced postural hypotension may be centrally medicated. Human Psychopharmacol 3:181-190.

O'Boyle CA, Harris D, Barry H, Cullen JH (1986): Differential effect of benzodiazepine sedation in high and low anxious patients in a "real life" stress setting. Psychopharmacology 88:226229.

Papp LA, Goetz R, Cole R, et al (1989): Hypersensitivity to carbon dioxide in panic disorder. Am $J$ Psychiatry 146:779-781.

Preskorn SH, Weller EB, Weller RA, Glotzbach E (1983): Plasma levels of imipramine and adverse effects in children. Am J Psychiatry 140:1332-1335.

Roose SP, Glassman AH (1989): Cardiovascular effects of tricyclic antidepressants in depressed patients with and without heart disease. J Clin Psychiatry Monogr 7:1-18.

Roth WT, Telch MJ, Taylor CB, Agras WS (1988): Autonomic changes after treatment of agoraphobia with panic attacks. Psychiatry Res 24:95-107.

Roth WT, Margraf J, Ehlers A, et al (1991): Stress test reactivity in panic disorder. Arch Gen Psychiatry (in press).

Sheehan DV, Ballenger JC, Jacobsen G (1980): Treatment of endogenous anxiety with phobic, hysterical, and hypochondriacal symptoms. Arch Gen Psychiatry 37:51-59.

Spitzer RL, Williams JB (1983): Structured Clinical Interview for DSM-III-Upjohn Version. New York State Psychiatric Institute, Biometrics Division.

Taylor CB, Hayward C, King R, et al (1990): Cardiovascular and symptomatic reduction effects 
of alprazolam and imipramine in patients with panic disorder: Results of a double-blind placebo controlled trial. J Clin Psychopharmacol 10:112-118.

Spitzer RL, Endicott J (1978): Schedule for Affective Disorders and Schizophrenia-Lifetime Version, 3rd ed. Biometrics Research Division, New York State Psychiatric Institute.

Truett J, Cornfield J, Kannel W (1967): A multivariate analysis of the risk of coronary heart disease in Framingham. J Chronic Dis 20:511-524.

Venables PH, Christie MJ (1980): Electrodermal activity. In Martin I, Venables PH (eds): Techniques in Psychophysiology. New York: Wiley, pp 3-67.

Warrington SJ, Padgham C, Lader M (1989): The cardiovascular effects of antidepressants. Psychol Med Monogr Suppl 16.

Zitrin CM, Klein DF, Woerner MG, Ross DC (1983): Treatment of phobias: I. Comparison of imipramine hydrochloride and placebo. Arch Gen Psychiatry 40:125-138. 